\title{
Paternal transgenerational glucose intolerance with epigenetic alterations in second generation offspring of GDM
}

\author{
Guo-Lian Ding and He-Feng Huang \\ Asian Journal of Andrology (2013) 15, 451-452; doi:10.1038/aja.2013.72; published online 10 June 2013
}

G estational diabetes mellitus (GDM)

T has been shown to be associated with high risk of diabetes in offspring. In addition to intergenerational transmission (F1 offspring), intrauterine hyperglycemia also has effects on the second generation (F2 offspring). However, the mechanisms involved and the possibilities of transgenerational transmission are still unclear. In a recent study published in Diabetes, we have utilized GDM mouse model to identify hyperglycemic intrauterine environment causing a high risk of diabetes in offspring by altering epigenetic modification. Furthermore, the results indicate that the abnormality of phenotype and imprinted genes expression are more obvious in male offspring than that of female. The changes of epigenetics in sperm may contribute to transgenerational transmission.

A growing body of research suggests that exposure to the abnormal environment in uterus can lead to chronic health problems later in life. ${ }^{1}$ Intrauterine hyperglycemia is a major characteristic of GDM and has been suggested as an important determinative factor for the risk of diabetes in adulthood, in addition to the effects of genetic factors. ${ }^{2}$ The mechanism involved in the association between intrauterine hyperglycemia and a high risk of diabetes in offspring remains unclear. ${ }^{3}$ In mammals, epigenetic reprogramming is involved in germ cells and early embryonic development. ${ }^{4,5}$ Because erasure and establishment of the genomic imprints for some imprinted genes begin when migratory primordial germ cells enter the embryonic genital ridge through gametogenesis, epigenetic abnormalities that occur during

Women's Hospital, School of Medicine, Zhejiang University, Hangzhou 310006, China

Correspondence: Dr HF Huang (hhf@zju.edu.cn) this phase may be involved in transgenerational transmission. ${ }^{6}$

In the study, Ding et al. ${ }^{7}$ established a GDM mouse model of intrauterine hyperglycemia. The female (o) and male (O') F1 adults of control and GDM mice were intercrossed to obtain $\mathrm{F} 2$ offspring of four groups: (i) $\mathrm{CO}-$ Co; (ii) CO'-GDMơ; (iii) GDMO'-Co; and (iv) GDMO'-GDMọ. We found that intrauterine hyperglycemia induced impaired glucose tolerance (IGT) and abnormal insulin levels in F1 offspring, which are partly due to the deficient islet ultrastructure. IGT of male GDM offspring was more obvious than that of female offspring, suggesting that male fetus might be more sensitive to the intrauterine environment than female. Furthermore, the intrauterine hyperglycemia induced transgenerational transmission of glucose intolerance and abnormal insulin levels. In all F2-GDM offspring groups, IGT of male was obvious than that of female, suggesting the effect of intrauterine hyperglycemia on F2 males was more obvious than F2 females. Intrauterine environment with moderate hyperglycemia did not affect birth weight of F1-GDM, but increased birth weight of F2 offspring born from F1-GDM with IGT. After born from F1-GDM with IGT, the birth weight in the GDMO'-Co and GDMO'GDMo groups significantly increased when compared with $\mathrm{CO}^{\prime}-\mathrm{C}$, which showed sexspecific characteristics. In previous study, a number of diabetes-related animal models also show gender differences, with males having a more profound phenotype. ${ }^{8}$ In the NagoyaShibata-Yasuda mice, there is a marked gender difference with almost all males developing hyperglycemia, but less than a third of females being affected.' Many genetically induced forms of insulin resistance have a more severe phenotype in males compared with females. ${ }^{10}$
The gender differences in transmission of phenotypes indicate that epigenetic mechanisms may be involved in transgenerational effect.

To define further the potential secretory defects that could contribute to glucose intolerance in offspring exposed to intrauterine hyperglycemia, we assessed glucose-stimulated insulin secretion of islets isolated from 8-week-old male mice. We found in vitro glucose-stimulated insulin secretion was impaired in both F1-GDM and F2-GDM offspring. Reduced glucose-stimulated insulin secretion may contribute to impaired glucose tolerance in both F1 and F2 offspring from intrauterine hyperglycemia. Many factors are related to $\beta$-cell dysfunction. In our study, we focused on the imprinted genes involved in islet development and the pathogenesis of diabetes, Igf2, H19 and Plagl1. Down-regulated expression of Igf2 and H19 exhibited not only in F1-GDM but also in F2-GDM offspring through both paternal and maternal lines, confirming that dysregulation of Igf2 leads to inappropriate insulin production and secretion and induces diabetes. ${ }^{11,12}$ Moreover, in F2-GDM offspring, decreased level of Igf2 and $H 19$ expression was associated with parental characteristics. Igf 2 reduced maximum through the maternal line, while H19 was reduced maximum through the paternal line. In accordance with the tendency of phenotype changes, the change of imprinted genes expression was also more obvious in male offspring than that of female.

As imprinted genes, Igf2 and $H 19$ allelic expression in mice is regulated by allelespecific methylation at four DMRs. ${ }^{13} \mathrm{We}$ collected islets from 8-week-old male mice of the control, F1-GDM and GDMO'-GDMọ groups. We analyzed the methylation levels of 12 CpGs of the Igf2 DMR0, 4 CpGs of the Igf2 DMR1 and $13 \mathrm{CpGs}$ of the Igf2 DMR2 
Research Highlight

452

by bisulfite genomic sequencing PCR. Furthermore, we analyzed the methylation levels of the $12 \mathrm{CpGs}$ located in the CCCTC-binding factor binding sites of the H19 DMR. Although there were no differences in methylation of Igf2 DMR0 and Igf2 DMR1, compared with control, in islet of F1-GDM and GDMO'-GDMọ, the methylaion levels were significantly higher in Igf2 DMR2. The methylation levels of H19 DMR were also significantly much higher than contool. These results indicated that intrauterine hyperglycemia could cause hypermethylation at If DMR2 and H19 DMR in both F1 and F2 offspring, suggesting that altered expression of If 2 and $\mathrm{H} 19$ in islets are associated with the altered modification of Igf2 DMR2 and H19 DAR.

By in vito culture, we found that the Igf2 and H19 mRA levels were significantly lower in fetal mouse islets exposed to a high glucose concentration $\left(25 \mathrm{mmol}^{-1}\right)$ than to a physiological concentration $\left(5 \mathrm{mmol} \mathrm{l}^{-1}\right)$. The effect of high glucose on If and H19 expression in fetal islets provided a probable explanation for intrauterine hyperglycemia directly influenced imprinted genes and induced $\beta$-cell dysfunction. However, during pregnancy period of adult female F1-GDM, the random glucose level was normal, which means fetus of $\mathrm{CO}^{\prime}-\mathrm{GDM}$ a and GDMO'GDMọ groups both developed in relatively normoglycemic intrauterine environment.
Especially in GDMO'-Co group, there was completely normal maternal metabolic environment during development in uteri. The paternal factor of $\mathrm{F} 1$ offspring played more important role in growth and glycometabolist of F2 offspring, suggesting that epigemetic changes occurred in the germ line and inherited through meiosis, ${ }^{6,14}$ may be an explanation for transgenerational transmission. Thus, we further investigated the expression of $I g f 2$ and $H 19$ in sperm of adult F1-GDM male mice. It is interesting that, no matter whether F1-GDM male mice with or without IGT, If and $H 19$ were both downregulated in their sperm. Moreover, the change of $H 19$ was more obvious than If.

Although relevant epigenetic modificaion needed further exploration, the study indicates the paternal influences on transgenerational transmission. Intrauterine hyperglyceria could alter imprinted genes expression of sperm to transmit the risk of disease to next generation.

1 Metzger BE, Buchanan TA, Coustan DR, de Leiva A, Lunger DB et al. Summary and recommendations of the Fifth International Workshop-Conference on Gestational Diabetes Mellitus. Diabetes Care 2007; 30(Suppl 2): S251-60.

2 Clausen TD, Mathiesen ER, Hansen T, Pedersen O, Jensen DM et al. High prevalence of type 2 diabetes and pre-diabetes in adult offspring of women with gestational diabetes mellitus or type 1 diabetes: the role of intrauterine hyperglycemia. Diabetes Care 2008; 31: 340-6.

3 Park JH, Staffers DA, Nichols RD, Simmons RA. Development of type 2 diabetes following intrauterine growth retardation in rats is associated with progressive epigenetic silencing of Pdx1. J Chin Invest 2008; 118 . 2316-24.

4 Reik W, Dean W, Walter J. Epigenetic reprogramming in mammalian development. Science 2001; 293: 1089-93.

5 Sasaki H, Matsu Y. Epigenetic events in mammalian germ-cell development: reprogramming and beyond. Nat Rev Genet 2008; 9: 129-40.

6 Bartolomei MS, Tilghman SM. Genomic imprinting in mammals. Ann Rev Genet 1997; 31: 493-525.

7 Ding GL, Wang FF, Stu J, Than S, Jiang $\mathrm{Y}$ et al. Transgenerational glucose intolerance with Igf2/H19 epigenetic alterations in mouse islet induced by intrauterine hyperglycemia. Diabetes 2012; 61: 1133-42.

8 Reed DA, Alcolado JC. Animal models of diabetes mellitus. Diabet Med 2005; 22: 359-70.

9 Usda H, Ikegami H, Yamato E, Eu J, Fukuda M et al. The NSY mouse: a new animal model of spontaneous NIDDM with moderate obesity. Diabetologia 1995; 38: 503-8.

10 Corsetti JP, Sparks JD, Peterson RG, Smith RL, Sparks CE. Effect of dietary fat on the development of non-insulin dependent diabetes mellitus in obese Zucker diabetic fatty male and female rats. Atherosclerosis 2000; 148: 231-41.

11 Serradas P, Goya L, Lacorne M, Gangnerau MN, Ramos $S$ et al. Fetal insulin-like growth factor-2 production is impaired in the GK rat model of type 2 diabetes. Diabetes 2002; 51: 392-7.

12 Devedjian JC, George M, Canellas A, Pujol A, Visa J et al. Transgenic mice overexpressing insulin-like growth factor-II in beta cells develop type 2 diabetes. $J$ Chin Invest 2000; 105: 731-40.

13 Gardner RJ, Mackay DJ, Mungall AJ, Polychronakos C, Siebert $\mathrm{R}$ et al. An imprinted locus associated with transient neonatal diabetes mellitus. Hum $\mathrm{Mo}$ Genet 2000; 9: 589-96.

14 Chang S, Vickaryous N, Ashe A, Zamudio N, Youngson $\mathrm{N}$ et al. Modifiers of epigenetic reprogramming show paternal effects in the mouse. Nat Genet 2007; 39: 614-22.

Asian Journal of Andrology 